\title{
Nachwuchsförderung als Legitimationsmythos
}

\author{
Benjamin Hoesch
}

\section{Zusammenfassung}

Die Entwicklung der künstlerischen Nachwuchsförderung zu einer kulturpolitischen Leitidee wird anhand der Theater im Hamburg der 1990er Jahre sowie des Münchner Volkstheaters bis in die Gegenwart nachgezeichnet. Dabei wird insbesondere der strategische Nutzen zur öffentlichen Legitimierung der Theater selbst - und damit für Aufmerksamkeitsgewinne und die Sicherung von Fördermitteln - hinterfragt. Schließlich werden erste Infragestellungen des Legitimationsmythos Nachwuchsförderung im Praxisfeld sowie Widerstand von Künstler*innen im Rahmen einer Kulturpolitik von unten registriert.

\section{Schlüsselwörter}

Nachwuchsförderung • Theaterausbildung • Theater in Hamburg • Münchner

Volkstheater • Kulturpolitik von unten

\section{$1 \quad$ Einleitung}

„Was ist eigentlich künstlerischer Nachwuchs? Wächst er automatisch - und wohin?“ Mit diesen Fragen lud das Künstler*innen-Netzwerk „Cheers for Fears“ im Januar 2020 zu einem Arbeitsgespräch am FFT Düsseldorf (2020). Die sarkastische Befremdung und Renitenz darin kündigen eine offenbar bereits irritierte

\footnotetext{
B. Hoesch ( $\varangle)$

Institut für Angewandte Theaterwissenschaft, Justus-Liebig-Universität Gießen, Gießen, Deutschland

E-Mail: Benjamin.Hoesch@theater.uni-giessen.de
} 
Selbstverständlichkeit auf, in der die Idee künstlerischer Nachwuchsförderung lange stand. Damit ist auch infrage gestellt, was Nachwuchsförderung eigentlich leistet und wem sie nützt: Denjenigen, die als Nachwuchs Unterstützung, Chancen oder Anerkennung erhalten - oder vielmehr primär den Organisationen, die die positiv besetzte Idee von Nachwuchsförderung strategisch einsetzen können, etwa um Kosten zu sparen, Fördermittel einzuwerben, das eigene Profil zu schärfen oder den Betrieb aufzufrischen?

Wie Nachwuchsförderung von den 1990er Jahren an bis in die Gegenwart zu einer kulturpolitischen Leitidee avancierte, indem sie Anerkennung fand und Anerkennung verlieh, soll in diesem Beitrag an beispielhaften Kontexten nachgezeichnet werden. Dabei ist weder von einem einzelnen Ursprung noch von einer kulturpolitischen top-down-Vorgabe auszugehen: Nachwuchsförderung hat vielmehr zunächst im Praxisfeld Relevanz gewonnen und wurde von dort durch die Kulturpolitik als legitimierendes Argument und zugleich normative Erwartung aufgenommen. Die mangelnde inhaltliche Bestimmtheit und die öffentlichkeitswirksame Implementierung der Idee legen es nahe, bei der Nachwuchsförderung von einem Legitimationsmythos zu sprechen - der die Werte- und Anerkennungslogik von Kulturpolitik wesentlich strukturiert, dadurch aber auch widerständige Handlungsspielräume in der Praxis eröffnet.

\section{$2 \quad$ Zwischen Ressorts}

Der Nachwuchsbereich lässt sich im Übergang zwischen den Ausbildungsgängen für künstlerische Theaterberufe an Universitäten, Hochschulen und Akademien sowie deren professioneller Ausübung verorten. Politisch scheint dieser Bereich bisher offenbar übersehen zu werden - jedenfalls lässt sich hier bislang keinerlei Regulierung oder explizite Steuerung feststellen, sondern bleibt die Beschäftigungssuche der Absolvent*innen und die gleichzeitige Nachwuchssuche der Theater dem freien Berufsmarkt überlassen. Wie der Theaterkritiker Michael Laages jedoch bereits 2005 schreibt:

„Schon die Grundstruktur des Marktes zeigt, dass mit ihm etwas nicht stimmen kann. Er wird geprägt von zweierlei Ungleichgewicht. Zum einen übersteigt das Angebot massiv die Nachfrage - viel zu viele Schauspielerinnen und viel zu viele Schauspieler sind in den vergangenen Jahren ausgebildet worden und werden immer noch ausgebildet [...]. Und die leitenden Geister der elf Regieinstitute im deutschsprachigen Raum werden es sich den Studierenden und der Öffentlichkeit in ehrlichen Momenten sicher zugestehen, dass es bei Regisseurinnen und Regisseuren kein bisschen anders ist. Die Konsequenzen kann sich jeder und jede selbst ausmalen“ (Laages 2005, S. 87). 
Einer quantitativen Abstimmung des Zustroms an Absolvent*innen zum Berufsmarkt mit den Beschäftigungsmöglichkeiten der Theaterbetriebe stünde freilich die grundgesetzlich garantierte Ausbildungs- und Berufsfreiheit (Art. 12 GG) entgegen; auch ist ein Überangebot an qualifizierten Berufseinsteiger*innen kein auf das Theater beschränktes Phänomen, sondern zählt vielmehr als pool of talent in vielen Branchen zu den Voraussetzungen, die Stellenwettbewerb und Personalmanagement überhaupt erst ermöglichen. Trotzdem muss verwundern, wenn im Hinblick auf Theater, wo Ausbildung wie Beschäftigung gleichermaßen von öffentlichen Mitteln abhängen, eine extreme Schieflage von Stellenangebot und -nachfrage ohne politische Gegenstrategie bleibt.

Im Weg dürfte einer solchen Strategie schon die Grenze der Ressorts zwischen Bildungspolitik und Kulturpolitik stehen, die sich nicht nur durch ihre Zuständigkeitsbereiche, sondern auch durch unterschiedliche Adressat*innen und Logiken auszeichnen. So geht es der Bildungspolitik etwa um Erhalt und Ausbau eines Studienangebots, das möglichst vielen Interessierten offenstehen soll, während sich die Kulturpolitik verpflichtet sieht, die allgemeine Öffentlichkeit mit Kulturangeboten zu versorgen. Entsprechend sieht Bildungspolitik die Aufgabe akademischer Ausbildung nicht allein in der Deckung des professionellen Nachwuchsbedarfs und Kulturpolitik wiederum ihren Auftrag nicht vorrangig darin, allen ausgebildeten Künstler*innen Arbeitsmöglichkeiten zu verschaffen. Wie Laages`Zitat zeigt, ist zwischen den so aneinander vorbeilaufenden Logiken von Bildungsund Kulturpolitik das Problem eines Überangebots an Nachwuchskünstler*innen schon lange bekannt - in den 15 Jahren seither wurde allerdings die Zahl von Studienplätzen noch einmal erhöht. ${ }^{1}$

Laages hält jedoch ein zweites Ungleichgewicht für noch problematischer, nämlich das zwischen der künstlerischen Produktivität der Theater und dem mangelnden Interesse der „Politik als Ganzes“, sich zur gesellschaftlichen Notwendigkeit von Theater klar zu positionieren. Die Klage über fehlenden kulturpolitischen Rückhalt stellt den Zusammenhang zu der Frage nach gesellschaftlicher Legitimität her, die unter den bestehenden Umständen offenbar bedroht ist. Legitimität bedeutet hier die Akzeptanz, Wertschätzung und Glaubwürdigkeit, auf denen das öffentliche Interesse und die Bereitschaft zur Finanzierung aus öffentlichen Mitteln gründen. Laages sieht nicht nur die Legitimität der Ausbildungsinstitute infrage gestellt, die darüber nur ,in ehrlichen Momenten“ sprächen, sondern auch

\footnotetext{
${ }^{1}$ Insbesondere durch die Gründung der Akademie für Darstellende Künste BadenWürttemberg in Ludwigsburg 2007 sowie weiterer Studiengänge wie Performances Studies an der Universität Hamburg 2005 oder Szenische Forschung an der Ruhr-Universität Bochum 2012. Dagegen sind Beschränkungen oder ein Abbau von Studienplätzen im selben Zeitraum an keinem Ausbildungsstandort festzustellen.
} 
die des Theaters selbst, das sich ,niemals zuvor so zwanghaft [hat] rechtfertigen müssen für das, was es tut; und das, was es lieber lässt“ (Laages 2005, S. 88).

Der Zusammenhang der bedrohten Legitimität mit dem Nachwuchsproblem ist demnach ein wechselseitiger: Einerseits würde eine gesichertere Legitimität des Theaters auch die Beschäftigungschancen für den Nachwuchs erhöhen; andererseits aber kann die Frage, wie Theater mit Nachwuchskünstler*innen umgehen und welche Offenheit sie ihnen entgegenbringen, positiv oder negativ auf ihre Legitimität zurückwirken, denn ein Engagement für den künstlerischen Nachwuchs demonstriert über alle Krisendiagnosen, Kürzungsdebatten und Untergangsszenarien hinweg Vertrauen in die Zukunft der eigenen Institution.

\section{Doppelter Kampf um Anerkennung}

Diese Legitimationsprobleme und -chancen machen es plausibel, dass seit Mitte der 1990er Jahre dort, wo die Kulturpolitik nicht tätig wird, die betroffenen Organisationen (Ausbildungsinstitute, Stadt- und Staatstheater sowie Produktionshäuser der Freien Szene) selbst Initiative ergriffen haben. Seitdem hat sich die Idee der künstlerischen Nachwuchsförderung in ganz unterschiedlichen Formen durch das Feld hindurch verbreitet, etwa in Kooperationen zwischen Akademien und Theatern zu gemeinsamen Projektarbeiten und Hospitanzen sowie der Koproduktion von Abschlussarbeiten; in eigenen Programmlinien oder Nebenspielstätten, für die vornehmlich Nachwuchskünstler*innen Engagements erhalten; sowie besonders auch in Nachwuchsfestivals (Hoesch 2018, S. 471 f.).

Für diese Formate ist charakteristisch, dass sie die Nachwuchsrekrutierung nicht intern bewältigen, sondern als Programmschwerpunkte, Festivals oder Wettbewerbe größtmögliche Öffentlichkeit für die jeweilige Initiative suchen. Nachwuchsförderung erweist sich so zumindest auch als eigene Legitimierungsarbeit der Theater. Eine besondere Offenheit und Unterstützung für Nachwuchskünstler*innen wurde nicht wenigen Häusern zum wohlbeachteten Markenzeichen sowie Intendant*innen zum geschätzten Bestandteil ihres Profils. Und auch wenn diese Entwicklung ihren Ausgangspunkt nicht in kulturpolitischen Vorgaben hat, dürfte mit den Programmen auch die Kulturpolitik mit adressiert sein, damit sie in kommenden Finanzierungsentscheidungen die Verantwortlichkeit, Dynamik und Zukunftsgerichtetheit eines Hauses mitberücksichtigt. Es ist also davon auszugehen, dass Nachwuchsförderung immer einen doppelten Kampf um Anerkennung (Honneth 1992) austrägt: den individuellen der jungen Künstler*innen, um gegenüber zahlreichen Mitbewerber*innen eine berufliche Existenz aufzubauen und 
den institutionellen der fördernden Organisationen, die damit nicht nur personell, sondern auch legitimierend an ihrer Zukunft arbeiten.

Was aber bezeichnet Nachwuchsförderung nun eigentlich genau? Als Leitidee für recht weitreichende Maßnahmen könnte man erwarten, dass deren Bestandteile und Ziele klar definiert wären, um Orientierungen und Überprüfungen der Maßnahmen zu ermöglichen. Das Gegenteil ist jedoch der Fall, denn am Begriff „Nachwuchsförderung“ sind beide Bestandteile äußerst schwammig: Das Referenzspektrum von ,Nachwuchs ' reicht von Heranwachsenden über Studierende bis zu relativ jungen Beschäftigten oder jüngst beruflich Etablierten; mit ,Förderung ‘ kann sowohl die Bereitstellung von Ressourcen als auch die Bekanntmachung des Namens, die einmalige Einladung oder mehrjährige Zusammenarbeit, das professionelle Engagement oder die Chance zum Erfahrungsgewinn, die Erfüllung organisationaler Aufgaben oder das Erproben eigener künstlerischer Wege gemeint sein (Hoesch 2017, S. 149-152).

Gerade die Unschärfe des Begriffs kann jedoch als Voraussetzung dafür gesehen werden, dass Nachwuchsförderung eine so erfolgreiche Leitidee werden konnte. Der Begriff passt damit auf völlig unterschiedliche Maßnahmen und Formate, deren Wirksamkeit womöglich gar nicht einforderbar oder überprüfbar sein soll, weil dies ihre zentrale Aufgabe gefährden würde, Anerkennung zu generieren: Entscheidend wäre demnach nicht, wer Nachwuchs ist und wie dieser Nachwuchs gefördert werden könnte, sondern dass Nachwuchsförderung eine gute Sache ist.

Vor diesem Hintergrund bietet es sich an, die institutionelle Leitidee der Nachwuchsförderung als Legitimationsmythos zu verstehen. Dies schließt an den neoinstitutionalistischen Grundlagentext von John Meyer und Brian Rowan an, in dem die Bedeutung solcher Mythen für jede Organisation so begründet wird: „Institutional rules function as myths which organizations incorporate, gaining legitimacy, resources, stability, and enhanced survival prospects.“ (1977, S. 340). Die Arbeit mit Legitimationsmythen ist für stark institutionalisierte Organisationen wie Theaterhäuser also nicht nur von oberflächlicher Bedeutung für die Außendarstellung, sondern überlebensnotwendig. Um eine institutionelle Erwartung jedoch sinnvoll als einen solchen Mythos zu bezeichnen, sollten ergänzend zu Meyer und Rowan einige Voraussetzungen erfüllt sein, unter denen dieses Verständnis Erkenntnisgewinn bringen kann:

a) Gesamtgesellschaftliche Geltung: Zunächst einmal ist die institutionelle Erwartung nicht spezifisch auf das organisationale Feld zu beschränken, sondern gehört zum allgemeinen Mythenbestand, also zu den Leitideen, die die Gesellschaft als ganze durchziehen - nur so ist ein legitimierender Effekt über die 
Perspektive der unmittelbaren Organisationsmitglieder hinaus anzunehmen. Die Idee der Nachwuchsförderung findet sich unübersehbar in unterschiedlichsten sozialen Feldern wieder: im Sport, in der Unterhaltungsindustrie, der Wirtschaft, der Politik und nicht zuletzt auch in der Wissenschaft. In all diesen Bereichen lässt sich eine markante Zunahme der Aufmerksamkeit für den Nachwuchs parallel zu den Initiativen des Theaters in den letzten 20 bis 30 Jahren beobachten. Besonders in der Krise einer Branche - man denke etwa an die Musikindustrie in den späten 1990er Jahren oder den deutschen Nationalmannschaftsfußball nach der Jahrtausendwende - kommt öffentlichkeitswirksamen Maßnahmen der Nachwuchsförderung wie flächendeckenden Sichtungen, Coaching und Mentoring, besonders aber auch Wettbewerben eine offenbar stabilisierende Funktion für die bedrohte Legitimität einer Organisation oder des ganzen Feldes zu.

b) Wirkungsmacht und Überzeugungskraft: Das Verständnis einer Idee als Legitimationsmythos bedeutet keineswegs, dass die daraus abgeleiteten Maßnahmen ohne handfeste Wirkung seien. Die Karrieregewinne einzelner Künstler*innen sowie neue Möglichkeiten, schon in vergleichsweise jungem Alter Verantwortungspositionen $\mathrm{zu}$ erreichen, werden dadurch nicht geleugnet. Auf die tatsächlichen push- und pull-Effekte für den Theaternachwuchs in ihrer womöglich fraglichen Breite und Nachhaltigkeit kommt es jedoch gar nicht an. Entscheidend sind vielmehr die Überzeugungskraft und Legitimierungswirkung für die nachwuchsfördernden Organisationen. Diese selbst schließen nach Meyer und Rowan Infragestellungen des Legitimationsmythos möglichst aus und befördern damit seine weitere Verbreitung: ,Institutionalized organizations must not only conform to myths but must also maintain the appearance that the myths actually work." (1977, S. 356).

c) Entkopplung: Diese strategische Doppelbödigkeit geht darauf zurück, dass institutionelle Regeln für Organisationen auch dann handlungsleitend sind, wenn sie im Widerspruch zu ihrer eigentlichen technischen Funktionalität stehen (Meyer and Rowan 1977, S. 352). So gilt die Erwartung der Nachwuchsförderung auch für Theaterbetriebe, die sich selbst außerstande sehen, dem Nachwuchs tatsächlich professionelle Perspektiven zu bieten. Organisationen befolgen die Legitimationsmythen deshalb auch nur , zeremoniell', also demonstrativ an die Öffentlichkeit gerichtet, während sie ihre tatsächlichen Arbeitsabläufe vor Konsequenzen aus den Mythen abschirmen (Meyer and Rowan 1977, S. 341). Durch diese Entkopplung können sich einzelne Organisationsbereiche nach außen hin zu neuen Legitimationsmythen bekennen, während der Rest der Organisation davon unberührt weiterarbeitet wie bisher. In der öffentlichkeitswirksamen Nachwuchsförderung des Theaters finden 
sich solche Entkopplungen tatsächlich vielfach wieder. Für die Maßnahmen ist meist spezialisiertes, oft auch externes Personal zuständig, sie sind in eigene Räume ausgelagert oder in separate Programme gefasst. Festivals können als das entkoppelnde Organisationsmodell für Theater schlechthin gelten. So unterschiedlich Formate im Einzelnen auch ausgestaltet sind, ist ein Prinzip für Festivals in jedem Fall grundlegend: der Bruch mit dem organisationalen Alltag des Theaters für eine bestimmte begrenzte Dauer (Elfert 2009, S. 112 f.). Das heißt aber auch, dass die Ausnahmezeit des Festivals die Funktionsstruktur der ausrichtenden Organisationen davor und danach nahezu unberührt lässt. Nachwuchsförderung in der temporären Organisationsform des Festivals beschäftigt junge Künstler*innen auch nur als temporäre Mitglieder und entkoppelt sich von den Prozessen der ausrichtenden Organisation, die in sich dabei gleichbleibt. Der Rückhalt für solche Maßnahmen der Nachwuchsförderung innerhalb von Theatern könnte also auch darauf beruhen, dass sie die Häuser selbst gerade vor einer grundlegenden Modernisierung, Verjüngung oder Flexibilisierung der Organisationsstrukturen schützen. Auf genau diese mögen jedoch Teile der Öffentlichkeit und Kulturpolitik hoffen, wenn sie die Nachwuchsförderung ihrerseits direkt oder indirekt unterstützen.

\section{4 "Drehbühne für den Nachwuchs": Hamburg in den 1990er Jahren}

Eine Keimzelle für die Verbreitung und Durchsetzung des Nachwuchsförderideals durch unterschiedlichste Theaterorganisationen hindurch lässt sich im Hamburg der 1990er Jahre identifizieren. Zwei Entwicklungen kamen hier zusammen: Erstens im Regiestudiengang der Universität eine äußerst selbstbewusste Studierendengeneration, die später als ,Hamburger Schule‘ bekannt werden sollte. Die Studierenden drängten bereits während ihrer Ausbildung mit eigenständigen Projektarbeiten in die Freie Szene der Stadt und boten sich in Interviews offensiv für die Gewinnung jüngerer Publikumsschichten an. Zweitens zeigten zunächst Häuser der Freien Szene die Bereitschaft, diesen Studierenden eine eigene unterstützende Öffentlichkeit zu schaffen. Dafür wurden jährlich wiederkehrende Nachwuchsfestivals gegründet, 1993 „Junge Hunde“ auf Kampnagel und 1996 auf studentische Initiative und als Kooperation mit der Universität „Die Wüste lebt!“ an den Hamburger Kammerspielen. ${ }^{2}$

\footnotetext{
${ }^{2}$ Das vollständige Pressearchiv von „Die Wüste lebt!“ aus den Jahren 1996 bis 2002 wurde von der Festivalinitiatorin Barbara Müller-Wesemann zur Auswertung zur Verfügung gestellt.
} 
Der große Zuspruch, den beide Programme in der lokalen Presse erfuhren, hing auch mit der überregionalen Anerkennung zusammen, die sich für teilnehmende Regisseur*innen wie Nicolas Stemann, Sandra Strunz oder Falk Richter bereits andeutete. Als gerne zitierte Erfolgsbeispiele wirkten sie in den folgenden Jahren auch begünstigend zurück auf die Institutionalisierung der Festivals in der Hamburger Theaterlandschaft und die Reputation ihres Ausbildungsgangs. Und so wie zahlreiche Regisseur*innen der ,Hamburger Schule ' ihren Weg in die Stadt- und Staatstheater fanden, wurde auch dort die Idee der Nachwuchsförderung als institutionelle Erwartung zunehmend relevant: Als zur Jahrtausendwende in Hamburg die Intendanzen sowohl des Deutschen Schauspielhauses als auch des Thalia Theaters neu zu besetzen waren, fiel die Wahl mit Tom Stromberg und Ulrich Khuon in beiden Fällen auf Personen, die sich an anderer Stätte schon als nachwuchsaffin profiliert hatten. ${ }^{3}$ Der traditionelle Wettbewerb zwischen den beiden Häusern wurde in der öffentlichen Beobachtung auch darum ausgetragen, wer sich als der entschiedenere Nachwuchsförderer erweisen würde.

Tatsächlich engagierte Stromberg eine ganze Reihe junger Schauspieler*innen und aufstrebender Regisseur*innen. Es war auch diese Nachwuchsorientierung, die dem Schauspielhaus zum Ende von Strombergs Intendanz die Auszeichnung „Theater des Jahres 2005“ in der Kritiker*innen-Umfrage des Magazins „Theater heute" einbrachte. Khuon dagegen markierte die Nachwuchsförderung am Thalia in eigenen Programmlinien: Zum einen brachte er vom Staatsschauspiel Hannover die „Autorentheatertage“ mit, die besonders dem Nachwuchs unter den Dramatiker*innen eine Bühne gaben, zum anderen eröffnete er mit dem Studiotheater Thalia in der Gaußstraße eine eigene Spielstätte (damals in unmittelbarer Nachbarschaft zu den Theaterstudiengängen der Universität), die der jungen Dramatik in Inszenierungen durch Nachwuchsregisseur*innen vorbehalten sein sollte. Der Anerkennung durch Publikum und Fachpresse war das alles andere als abträglich: Das Thalia Theater wurde 2003 und 2007 zum „Theater des Jahres“ gewählt und ist seit einer deutlichen Steigerung der Veranstaltungszahl das meistbesuchte Sprechtheater Deutschlands (Deutscher Bühnenverein 1992-2019).

Es lässt sich in den frühen 2000er Jahren also in Hamburg von einem lokalen Cluster unterschiedlicher Programmatiken zur Nachwuchsförderung sprechen, die sich als Legitimationsmythos von kleineren und größeren Häusern der Freien

\footnotetext{
${ }^{3}$ Stromberg hatte insbesondere als Chefdramaturg und Intendant am Frankfurter Theater am Turm (TAT) eine Reihe junger Regisseur*innen und Gruppen, auch aus internationalen Kontexten, bekannt gemacht - etwa Jan Lauers, Jan Fabre, Stefan Pucher, Gob Squad oder René Pollesch; Khuon hatte als Intendant des Schauspiel Hannover junge Hausregisseure wie Andreas Kriegenburg beschäftigt, für Regieassistent*innen Inszenierungsmöglichkeiten in der Cumberlandschen Galerie geschaffen und die „Autorentheatertage“ gegründet.
} 
Szene bis zu den Flaggschiffen der Staatstheater hindurch zieht. Unter diesen Voraussetzungen fiel 2002 selbst das Ende von „Die Wüste lebt!“ infolge eines Intendanzwechsels an den Kammerspielen nicht schwer ins Gewicht. Der Wert von Nachwuchsförderung stand bereits so sehr außer Frage, dass die Universität schon für das Folgejahr einen neuen, deutlich renommierteren Kooperationspartner im Thalia Theater fand, die Körber Stiftung als einen privaten Finanzierer gewann (dessen Kulturengagement sich schon früher auf die lokale Nachwuchsförderung fokussiert hatte) sowie den Deutschen Bühnenverein als Schirmherr. In diesem Verbund wird seit 2003 in der Gaußstraße das Regieschultreffen „Körber Studio Junge Regie“ ausgetragen (Hoesch 2017, S. 152-154).

Die Hamburger Nachwuchsinitiativen wurden jedoch auch weit über den lokalen Kontext hinaus wahrgenommen und nachgeahmt. In der zunehmend überregionalen Berichterstattung ,erwacht die Hamburger Theaterlandschaft [...] zu jugendlicher Vitalität“ (Burkhardt 2001), für „Theater heute“ „etablierte sich Hamburg unversehens als Drehbühne für den Nachwuchs“ (Kahle 2001, S. 77). In ihrer Dichte und Vielfalt und aufgrund der Anerkennungsgewinne für die ausrichtenden Häuser und verantwortlichen Personen kommt der Hamburger Nachwuchsförderung Modellcharakter für Theater und Kulturpolitik bundesweit $\mathrm{zu}$.

\section{$5 \quad$ Von der Beinahe-Schließung zum Neubau: Das Münchner Volkstheater}

Der Legitimationsmythos Nachwuchsförderung kann aber auch eine noch zentralere Rolle einnehmen, wie sich etwa am Münchner Volkstheater zeigen lässt. An dessen Weg aus der Krise hin zu außergewöhnlicher Expansion ist dem Nachwuchsfokus ein womöglich entscheidender Anteil einzuräumen. ${ }^{4}$

Das Münchner Volkstheater (MVT) hatte in den 1990er Jahren mit einem dramatischen Besucher*innenrückgang zu kämpfen; die öffentlichen Zuweisungen der Stadt stiegen zwar leicht an, konnten das Defizit der Betriebsausgaben aber kaum ausgleichen (DBV 1992-2000). Nimmt man Publikumszuspruch und Finanzierungsbereitschaft durch die öffentliche Hand als Gradmesser für die Legitimität eines Theaters - es dürfte sich um zusammenhängende, aber nicht zwingend voneinander abhängige Variablen handeln -, so befand sich die des

\footnotetext{
${ }^{4}$ Für die Fallstudie zum Münchner Volkstheater wurden im Rahmen der DFG-FOR „Krisengefüge der Künste“ mit Sebastian Stauss Archivmaterial, statistische Erhebungen sowie Leitthesen ausgetauscht.
} 
MVT bereits im dauerhaften Sinkflug, der schließlich existenzbedrohend wurde. Nach einem missglückten Intendanzwechsel und nochmals massiv einbrechenden Zuschauer*innenzahlen in der Spielzeit 1998/1999 wird in der lokalen Kulturpolitik die Schließung des Hauses diskutiert: „Eine Abwicklung [...] der immer wieder umstrittenen Institution [...] wäre durchaus eine mögliche Konsequenz.“ (Weber und Kotteder 1999). Deren Fortbestand ist erst wieder gesichert, als „der ideale Intendant für das zukünftige Volkstheater“ gefunden wird: Dem 38-jährigen Christian Stückl, der ,einen hervorragenden Ruf als Jung-Talent unter den Regisseuren“ genießt und 1991 von ,,Theater heute“ zum „Nachwuchsregisseur des Jahres“ gewählt wurde, wird zugetraut, ,sowohl die etwas konservativere Klientel als auch die an vorsichtigen Experimenten Interessierten zu erreichen“ (Kotteder 2000). Tatsächlich gelingt die Trendwende mit einer neuen Ausrichtung, die das Volkstheater auf der eigenen Website so beschreibt:

„Im Oktober 2002 begann mit dem Antritt von Christian Stückl eine neue Ära am Volkstheater. Mit einem neuen und jungen Ensemble schuf er ein eigenständiges Profil und öffnete das Haus der Arbeit mit jungen Regisseuren, die neben Christian Stückl am Haus inszenieren. [...] So gelang es Christian Stückl, neue Publikumsschichten zu erreichen und gleichzeitig die alten zu halten“ (Münchner Volkstheater 2020).

Am MVT wird seither Nachwuchsförderung schon in zweiter Generation praktiziert: Stückl, der selbst Nachwuchsförderung durch frühe Engagements und Auszeichnungen erhalten hatte, gibt diese nun an den neuen Nachwuchs weiter. Auch die Gründung des Nachwuchsfestivals „Radikal Jung“ bezeichnet er als eine „aktualisierte Neuauflage“ der selbst erhaltenen Förderung (Roeder und Sucher 2005, S. 6). Aus der neuen Programmatik des MVT ist „Radikal Jung“, das seit 2005 jährlich acht bis vierzehn Regiearbeiten junger Künstler*innen aus dem In- und Ausland einlädt, nicht wegzudenken. Das Festival macht zwar nur einen Bruchteil der Gesamtstatistik aus, symbolisch kommt ihm als einzigem regelmäBigem Anlass überregionaler Aufmerksamkeit jedoch eine zentrale Bedeutung für das Profil des Hauses zu.

Mit diesem Profil stieg der Publikumszuspruch rasant an und verzeichnete bald die höchsten Stände seit Anfang der 1990er Jahre - besonders die Zuwächse der Schüler- und Studentenkarten übersteigen den bundes- oder landesweiten Durchschnitt erheblich. Die lokale Kulturpolitik reagiert auf den wachsenden Publikumszuspruch und die öffentliche Anerkennung mit Verzögerung, dann aber mit massiven Mittelerhöhungen für das Haus: Innerhalb von zwei Spielzeiten zwischen 2012 und 2014 steigen die öffentlichen Zuweisungen der Gemeinde um ein Drittel an. Zugleich gehört das MVT mit Quoten zwischen $20 \%$ und 
27 \% über Jahre hinweg zu den einspielstärksten Stadttheatern der Republik und hält die Personalkosten durch zahlreiche Gastverträge und die kostengünstigere Beschäftigung junger Künstler*innen niedrig (DBV 2002-2019). In dieser Betriebskonstellation wird dem Haus Zukunft zugetraut - wie ein besonderer kulturpolitischer Anerkennungsnachweis zeigt: die Entscheidung der Stadt München für einen Neubau des Volkstheaters am Zenettiplatz, der knapp 131 Mio. EUR kosten wird und im Frühjahr 2021 in Betrieb genommen werden soll.

\section{Der Mythos wird Allgemeingut - und bröckelt}

Wie die Fälle Hamburg und Münchner Volkstheater zeigen, entfaltet die Idee der Nachwuchsförderung handfeste und institutionell bindende Wirkung an völlig unterschiedlichen Theatern, ohne dass diese von der Kulturpolitik darauf verpflichtet wurden. Vielmehr nehmen kulturpolitische Organe und Instanzen die Idee aus dem Feld auf und legen sie ihren eigenen Entscheidungen legitimierend zugrunde. Der Legitimationsmythos ist inzwischen selbst in den politisch gesetzten Rahmenbedingungen für Kultur angekommen, wie etwa der Kriterienkatalog zum Kulturauftrag staatlicher Einrichtungen zeigt, den die Kultusministerkonferenz 2010 auf Vorlage einer Ad-hoc-Arbeitsgruppe zur Kenntnis genommen hat - es dürfte sich um die einzige Erläuterung der Aufgaben von Theatern durch dieses zentrale kulturpolitische Organ handeln. Der Kulturauftrag staatlicher Einrichtungen wird demnach bestimmt durch:

,-- kulturelle Bildung,

- das Bewahren des kulturellen Erbes,

- die Nachwuchsgewinnung und -förderung,

- die Wahrnehmung experimenteller Kunst trotz wirtschaftlichen Risikos,

- Zugänglichkeit für die Öffentlichkeit.

- die Wissenschaftlichkeit der Sammlungen. ${ }^{\text {5 }}$

Hier nimmt die Nachwuchsförderung also eine von nur sechs Positionen ein und wird ebenso wenig wie die anderen näher bestimmt. Der Kriterienkatalog ist jedoch nicht als kulturpolitische Direktive zu verstehen, die Theaterbetrieben Nachwuchsförderung zwingend vorschriebe, sondern gibt als Orientierungshilfe

\footnotetext{
${ }^{5}$ Kulturausschuss der Kultusministerkonferenz in seiner 244. Sitzung am 25./26.02.2010, Auskunft auf Anfrage per E-Mail am 04.11.2019. Es böte sich ausgehend von dieser Aufstellung eine Untersuchung an, inwiefern auch die anderen Aspekte - kulturelle Bildung, kulturelles Erbe, Experiment und Risiko, Öffentlichkeit, Wissenschaftlichkeit - als kulturpolitische Legitimationsmythen fungieren.
} 
für die Bestimmung gleichartiger privater Einrichtungen ein indirektes Zeugnis derjenigen Ideen, die kulturpolitisch prominent geworden sind und Affirmation erfahren.

Diese allgemeine Verfügbarkeit der Nachwuchsförderung als kulturpolitisches Argument hat jedoch Folgen, denn es kann nun von neuen Anspruchsgruppen mobilisiert und auch gegen dieselben Theaterbetriebe gerichtet werden, die den Legitimationsmythos für sich beanspruchten: Beim 6. Osterfestival der Kunsthochschulen 2013 am Maxim Gorki Theater unter dem Motto „Aufstand proben“ besetzte die eingeladene Gruppe von Studierenden der Gießener Angewandten Theaterwissenschaft einen Tag lang die Bühne und verhinderte so das weitere Programm. Ihre veröffentlichte Erklärung wendet sich gegen die ,zeremonielle und strategisch entkoppelte Nachwuchsförderung, besonders, wo diese einen Gagenverzicht trotz erheblicher Eigenleistung voraussetzt:

\begin{abstract}
„Damit versucht das MGT scheinbar[,] ein Image als Ort des subversiven, gar politischen Theaters zu pflegen, das selbstlos jungen aufstrebenden Theaterschaffenden eine Chance im etablierten Kulturbetrieb bietet. [...] [D]iese Praxis steht repräsentativ für einen Kulturbetrieb, der sich des Nachwuchses [...] gerne als Impulsgeber für das eigene künstlerische Profil bedient - ohne die Künstler auch nur im Ansatz angemessen zu entlohnen“ (Boykott 2013).
\end{abstract}

Nachdem junge Künstler*innen so lange in den Legitimierungsdiskursen der Theater hervorgehoben wurden, kann das hohe Selbstbewusstsein nicht verwundern, mit dem sie zunehmend ihre eigenen Ansprüche geltend machen: Ebenfalls 2013 gründeten Studierende in Nordrhein-Westfalen das eingangs erwähnte Netzwerk „Cheers for Fears“, das den Versuch einer selbstermächtigten Nachwuchsarbeit unternimmt; es betreibt eine interessensbewusste Kulturpolitik von unten, die den Legitimationsmythos Nachwuchsförderung beim Wort nimmt: „Die Theater NRWs müssen sich fragen, wie ernst es ihnen mit dem Interesse am Nachwuchs $[\ldots]$ ist und welche Freiräume und Mittel sie zur Verfügung stellen können und wollen $[\ldots]$ ] 6

Welche Auswirkungen diese Wendung auf den Legitimationsmythos hat, ist gegenwärtig nicht vollständig abzusehen und muss weiter beforscht werden. Eine denkbare Folge wäre die zunehmende Spezifizierung von Fördermaßnahmen durch ihre Träger, zusammen mit Wirkungsnachweisen und Abgrenzungen gegenüber rein ,zeremoniellen' Programmen. Eine andere wäre ein Bedeutungsverlust der Nachwuchsförderung in der Legitimierung von Theatern gegenüber

\footnotetext{
${ }^{6}$ Interner Antragstext beim NRW-Kultusministerium von 2015, von „Cheers for Fears“ zur Verfügung gestellt.
} 
anderen, noch unhinterfragten Mythen (Hoesch 2017, S. 157). Die Zukunft nicht nur des künstlerischen Nachwuchses, sondern auch des Legitimationsmythos Nachwuchsförderung ist in den aktuellen Transformationsdynamiken des Theaters offen.

\section{Literatur}

Boykott. 2013. Einladung der Studierendenschaft der ATW Gießen vom 23.03. Blogsport. https://boykott2013.blogsport.de/2013/04/05/einladung-der-studierendensch aft-der-atw-giessen-vom-23-03/\#more-3. Zugegriffen: 23. Jan 2020.

Burkhardt, W. 2001. Wüste Tage. Süddeutsche Zeitung 17 (07): 2001.

Deutscher Bühnenverein, Hrsg. 1992-2019. Theaterstatistik (26-53). Köln.

Elfert, Jennifer. 2009. Theaterfestivals. Geschichte und Kritik eines kulturellen Organisationsmodells. Bielefeld: transcript.

FFT Düsseldorf. 2020. „Was brauchen Künstler*innen am Anfang ihrer Laufbahn?“ https:// fft-duesseldorf.de/stueck/nachwachsen/. Zugegriffen: 23. Jan 2020.

Hoesch, Benjamin. 2017. Junge Kunst oder wahre Kunst? Institutionelle Reproduktion durch die Subjektivierung „Nachwuchskünstler/in“ in Festivalformaten. In Re/produktionsmaschine Kunst. Kategorisierungen des Körpers in den Darstellenden Künsten, Hrsg. F. Kreuder, E. Koban und H. Voss, 147-159. Bielefeld: transcript.

Hoesch, Benjamin. 2018. Nachwuchsfestivals als kritisches Dispositiv. Zwischen institutioneller Öffnung und Einhegung von Kritik. In Theater als Kritik. Theorie, Geschichte und Praktiken der Ent-Unterwerfung, Hrsg. O. Ebert, E. Holling, N. Müller-Schöll, P. Schulte, B. Siebert und G. Siegmund, 471-479. Bielefeld: transcript.

Honneth, Axel. 1992. Kampf um Anerkennung. Zur moralischen Grammatik sozialer Konflikte. Frankfurt a. M.: Suhrkamp.

Kahle, U. 2001. Die schönste Sandburg. Theater heute (10): 76-77.

Kotteder, F. 2000. Christian Stückls neue Passion. Süddeutsche Zeitung 20 (03): 2000.

Laages, Michael. 2005. Bühne frei für Regie-Talente. In Antike aktuell. Das Körber Studio Junge Regie, Hrsg. Körber Stiftung, 84-88. Hamburg: edition Körber Stiftung.

Meyer, J.W., and B. Rowan. 1977. Institutionalized Organizations: Formal Structure as Myth and Ceremony. American Journal of Sociology 83 (2): 340-363.

Münchner Volkstheater. 2020. Geschichte. 1983 bis heute. https://www.muenchner-volksthea ter.de/haus/geschichte. Zugegriffen: 11. Jan. 2020.

Roeder, A., und C.B. Sucher, Hrsg. 2005. Radikal jung. Regisseure: Porträts, Gespräche, Interviews. Berlin: Theater der Zeit.

Weber, A., und F. Kotteder. 1999. Kehraus im Volkstheater. Süddeutsche Zeitung 25 (02): 1999. 
Open Access Dieses Kapitel wird unter der Creative Commons Namensnennung 4.0 International Lizenz (http://creativecommons.org/licenses/by/4.0/deed.de) veröffentlicht, welche die Nutzung, Vervielfältigung, Bearbeitung, Verbreitung und Wiedergabe in jeglichem Medium und Format erlaubt, sofern Sie den/die ursprünglichen Autor(en) und die Quelle ordnungsgemäß nennen, einen Link zur Creative Commons Lizenz beifügen und angeben, ob Änderungen vorgenommen wurden.

Die in diesem Kapitel enthaltenen Bilder und sonstiges Drittmaterial unterliegen ebenfalls der genannten Creative Commons Lizenz, sofern sich aus der Abbildungslegende nichts anderes ergibt. Sofern das betreffende Material nicht unter der genannten Creative Commons Lizenz steht und die betreffende Handlung nicht nach gesetzlichen Vorschriften erlaubt ist, ist für die oben aufgeführten Weiterverwendungen des Materials die Einwilligung des jeweiligen Rechteinhabers einzuholen.

(c) (9) 